\title{
THE AUTHORS
}

- Galina A. Bazilevskaya (LPI, Nuclear Physics and Astrophysics Division, Russia), specialist in solar-terrestrial physics with a keen interest in cosmic rays generation, propagation and modulation in the heliosphere and atmosphere.

- Rasmus Benestad (MET Norway, Oslo, Norway), physicist and specialist in climate analysis, statistics and sun-climate connections.

- Roxana Bojariu (National Meteorological Administration, Bucharest, Romania), specialist in climate variability and predictability, with a focus on North Atlantic/Arctic Oscillation.

- Jaša Čalogović (Hvar Observatory, University of Zagreb, Croatia), specialist in solar-terrestrial connections (e.g. cosmic ray-cloud link) and space weather.

- Thomas von Clarmann (Karlsruhe Institute of Technology, Karlsruhe, Germany), specialist in remote sensing and stratospheric chemistry and physics.

- Thierry Dudok de Wit (University of Orléans, France), solar-terrestrial physicist with a keen interest in the statistical analysis of solar spectral variability

- Ilaria Ermolli (INAF, Osservatorio Astronomico di Roma, Italy), specialist in solar magnetism, with a focus on its effects on irradiance variations.

- Patrizia Francia (University L'Aquila, Italy), magnetospheric physics, ULF waves, polar cap activity.

- Bernd Funke (IAA-CSIC, Granada, Spain), specialist in middle atmosphere science and satellite data analysis, with a particular interest in particle-induced solar variability.

- Hans Gleisner (Danish Meteorological Institute), specialist on retrieving climate data from GPS radio occultation measurements.

- Margit Haberreiter (PMOD/WRC, Switzerland), solar physicist with strong interest in the radiative transfer in the solar atmosphere, and the causes and consequences of solar variability.

- Joanna Haigh (Imperial College London, UK), specialist in atmospheric radiative transfer and radiative-dynamical coupling.

- Arnold Hanslmeier (University of Graz, Austria), specialist in solar cycle variation studies and space weather; has published several books for specialists and non-specialists in the fields of astrophysics.

(C) EDP Sciences 2015 
- Giles Harrison (University of Reading, UK), specialist in atmospheric electricity, and novel meteorological instrumentation.

- Sverre Holm (Dept. of informatics, University of Oslo, Norway), specialist on spectral estimation.

- Maarit J. Käpylä (Aalto University, Finland), solar dynamo theory and modelling of turbulent magnetoconvection.

- Ingo Kirchner (Freie Universität Berlin, Institute for Meteorology, Germany), climate modeller and meteorologist, with a special interest in climate dynamics and it's driving processes.

- Natalie Krivova (MPS - Göttingen, Germany), specialist in solar variability, solar irradiance and solar influence on Earth's climate

- Markus Kunze (Freie Universität Berlin, Germany), specialist in middle atmosphere data analysis and modeling.

- Erkki Kyrölä (Finnish Meteorological Institute, Helsinki, Finland), specialist in radiative transfer, remote sensing and Earth observation, with a focus on the middle atmosphere.

- Benjamin A. Laken (University of Oslo, Norway), a Physical Geographer, with a focus on applying modern computer analysis methods to investigate climate processes and impacts.

- Mai Mai Lam (Department of Meteorology, University of Reading, UK), solar-terrestrial physicist with expertise in radiation belts and solar windtroposphere coupling.

- Ulrike Langematz (Freie Universität Berlin, Berlin, Germany), specialist in modeling chemistry-climate interactions in the lower and middle atmosphere and solar influence on climate.

- Maria Carmen Llasat (University of Barcelona, Barcelona, Spain), specialist on meteorology and climatology, and natural hazards.

- Jean Lilensten (IPAG - Grenoble, France), specialist of the planetary upper atmospheres (thermospheres and ionospheres) and space weather.

- Franz-Josef Lübken (Director of IAP, Kühlungsborn, Germany), specialist in ground-based and in-situ measurements in the polar mesosphere/lower thermosphere.

- Katja Matthes (GEOMAR \& CAU Kiel, Germany), specialist in modeling solar influence on climate, with a focus on stratosphere-troposphere-ocean coupling. 
- Amanda Maycock (University of Cambridge, UK), specialist in composition climate interactions, with a focus on stratosphere-troposphere interaction.

- Irina A. Mironova (St. Petersburg State University, Department of Earth Physics, Russia), specialist in solar-terrestrial physics with a keen interest in solar cosmic ray propagation and their effects into polar atmosphere.

- Stergios Misios (Aristotle University of Thessaloniki, Greece), specialist in modelling solar-climate linkages.

- Dann Mitchell (Atmospheric physics, Oxford University, UK), specialist on the stratospheric polar vortex.

- Christian Muller (B.USOC- Brussels, Belgium), data manager of the SOLAR payload on the International Space Station and ICTP expert on natural forcings on climate.

- Kalevi Mursula (Oulu University, Finland), space climate, solar wind and heliospheric magnetic field, long-term solar effects to near Earth space and climate.

- Donal Murtagh (Chalmers University of Technology, Göteborg, Sweden), specialist in remote sensing and Earth observation techniques.

- Keri Nicoll (University of Reading, UK), specialist in atmospheric measurements and instrumentation with a focus on solar-terrestrial effects on clouds and atmospheric electricity.

- Tine Nilsen (Applied mathematics, The Arctic University of Norway), specialist on statistical analysis of paleoclimatic reconstruction and climate model data.

- Colin Price (Tel Aviv University, Israel), specialist in atmospheric electricity, thunderstorms, lightning and climate change.

- Kerstin Prömmel (Freie Universität Berlin, Institute for Meteorology, Germany), climate modeller and meteorologist, with a special interest in palaeoclimate and decadal climate variability.

- Craig J. Rodger (Department of Physics, University of Otago, New Zealand), specialist on the detection, analysis and impact of energetic electron precipitation into the atmosphere.

- Eugene Rozanov (Institute of Atmospheric and Climate Science, ETHZ and PMOD/WRC, Switzerland), specialist in the climate and atmospheric chemistry modelling.

- Michael Rycroft (University of Bath and CAESAR Consultancy, UK), specialist in the global electric circuit, with a broad range of interests in atmospheric and space science. 
- Kristoffer Rypdal (Applied mathematics, The Arctic University of Norway), specialist on the mathematics and statistics of climate variability.

- Martin Rypdal (Applied mathematics, The Arctic University of Norway), specialist on the mathematics of climate and finance.

- Hauke Schmidt (Max Planck Institute for Meteorology, Germany), climate modeller specialised in effects of natural and anthropogenic forcings, and atmospheric vertical coupling.

- Werner Schmutz (PMOD/WRC, Switzerland), an astrophysicist who is investigating the solar irradiance and its impact on the terrestrial climate.

- Annika Seppälä (Finnish Meteorological Institute, Finland \& British Antarctic Survey, United Kingdom), specialist in solar and magnetospheric particle precipitation impacts on the middle atmosphere chemistry and dynamics, satellite observation expert.

- Sami K. Solanki (Max Planck Institute for Solar System Research, Germany), solar and heliospheric physics, solar magnetism and Sun-Earth relations

- Timofei Sukhodolov, (Institute of Atmospheric and Climate Science, ETHZ and PMOD/WRC, Switzerland), specialist in the modelling of atmospheric chemistry.

- Eija I. Tanskanen (Finnish Meteorological Institute, Finland), geomagnetic activity and its solar and solar wind sources such as high-speed streams and Alfvén waves.

- Peter Thejll (Climate and Arctic Research, Danish Meteorological Institute). Investigated Sun-climate link, now specialising in statistics of extreme weather and climate events, and earth observations.

- Rémi Thiéblemont (GEOMAR Helmholtz Centre for Ocean Research Kiel, Germany), specialist in middle atmosphere dynamics and its influence on climate.

- Matthew Toohey (GEOMAR Helmholtz Centre for Ocean Research Kiel, Germany), specialist in the climate impacts of major volcanic eruptions.

- Kleareti Tourpali (Aristotle University of Thessaloniki, Greece), atmospheric physicist with expertise in ozone-climate interactions and solar activity effects, specialist in the atmospheric environment and climate.

- Ricardo M. Trigo (Universidade de Lisboa, Portugal), specialist in climate variability, climate extremes and climate change mechanisms.

- Ilya Usoskin (Sodankylä Geophysical Observatory, Finland), cosmic rays, ground level enhancements, grand solar maxima and minima. 
- José M. Vaquero (Universidad de Extremadura, Spain), physicist with a keen interest in the reconstruction of solar activity from historical documents.

- Astrid Veronig (University of Graz, Austria), solar physicist with research focus on flares, coronal mass ejections and their impact on the Earth's space weather.

- Pekka T. Verronen (Finnish Meteorological Institute, Finland), specialist on middle atmospheric physics and chemistry, and effects of solar energetic particle precipitation.

- Yoav Yair (Interdisciplinary Center, Herzliya, Israel), An atmospheric physicist specialising in thunderstorms and lightning on Earth and other planets. 